to chromosome $15 \mathrm{q} 14$, similar to that involved in families with juvenile myoclonic epilepsy. An autosomal recessive mode of inheritance with heterogeneity was suggested. (Neubauer BA, Fiedler B, Himmelhein B, et al. Centrotemporal spikes in families with rolandic epilepsy. Linkage to chromosome 15q14. Neurology Dec 1998,51:1608-1612). (Reprints: Dr Bernd A Neubauer, Dept of Neuropediatrics, University of Kiel, Schwanenweg 20, 24105 Kiel, Germany).

COMMENT. Both benign rolandic epilepsy, a common partial, idiopathic epilepsy syndrome, and juvenile myoclonic epilepsy, a generalized idiopathic syndrome, have been linked genetically to chromosome 15q14.

\title{
NEONATAL DISORDERS
}

\section{OUTCOME OF NEONATAL CEREBRAL INFARCTION}

Antenatal and perinatal factors, early clinical signs, electroencephalograms (EEG), and magnetic resonance imaging (MRI) findings were compared with neurodevelopmental outcome in 24 infants with neonatal cerebral infarction followed at the Dept of Paediatrics, Hammersmith Hospital, London, UK. Infarcts defined by MRI involved a major cerebral vessel in 19 and borderzones in 5. Duration of follow-up ranged from 15 months to 5 years. Of 7 (29\%) infants with abnormal neuromotor outcome, 5 were hemiplegic and 2 showed asymmetry of tone or function. None developed seizures. Adverse antenatal factors, present in $11(46 \%)$, perinatal continuous decelerations below 90 with slow recovery in 14 , meconium staining in 11 , cord blood $\mathrm{pH}<7.1$ in 2 , and Apgar $<5 / 1 \mathrm{~min}$ in 5 were not related to outcome. Abnormal signs on neonatal neurologic exam, chiefly hypotonia, were poor prognostic indicators. Both EEG and MRI were predictors of abnormal outcome. Abnormal neonatal EEG background was associated with later hemiplegia whereas epileptic discharges were not predictive. MRI showing involvement of hemispheres, basal ganglia, and internal capsule, but not one or two of these regions, tended to develop hemiplegia or asymmetry of tone. Concomitant thalamic involvement did not increase the risk of poor outcome. (Mercuri E, Rutherford M, Cowan F et al. Early prognostic indicators of outcome in infants with neonatal cerebral infarction: a clinical, electroencephalogram, and magnetic resonance imaging study. Pediatrics Jan 1999;103;39-46). (Reprints: Dr Eugenio Mercuri, Dept of Paediatrics, Hammersmith Hospital, Du Cane Rd, London W12 OHN, UK).

COMMENT. Neonatal EEG and MRI findings may be predictive of later outcome and development of hemiplegia in infants born with cerebral infarcts. Abnormal antenatal and perinatal factors and neonatal hypotonia fail to identify infants with a poor prognosis.

\section{ANTE/INTRAPARTUM RISKS OF NEONATAL ENCEPHALOPATHY}

The role of antepartum and intrapartum factors in the etiology of neonatal encephalopathy (NE) in 164 term infants was investigated in a Western Australian case-control study, with 400 randomly selected controls. The prevalence of NE was $3.8 / 1000$ term live births, with a $9.1 \%$ case fatality. The features of NE included seizures, abnormal tone, apneas, feeding difficulties, abnormal consciousness, and ventilatory support. Independent risk factors before conception and in the antepartum period included lower socioeconomic status, family history of seizures or other neurologic disease, conception after infertility treatment, maternal thyroid disease, severe pre-eclampsia, bleeding during pregnancy, viral illness, 